Recibido: 19-noviembre-2013

Aceptado: 26-noviembre-2013

\title{
LA POLÍTICA PÚBLICA Y EL TRANSPORTE MULTIMODAL: EJES DE DESARROLLO E INTEGRACIÓN REGIONAL
}

JHON O. USIÑA P. CETIF 


\section{RESUMEN}

La importancia estratégica que tiene la política pública en cuanto a la decisión de inversión en infraestructura para el servicio del transporte -pero no del transporte conocido comúnmente- sino aquel transporte multimodal; planificado y organizado, aquel que potencia el desarrollo local y fomenta la integración de las actividades económicas que se realizan en un país, es un asunto trascendental para afianzar el crecimiento de las economías de las naciones de hoy.

El tema de déficit de infraestructura para el transporte multimodal ha sido cuestión de decisión política, más que cuestiones de financiamiento de dichas obras; esto por razones antagónicas al cambio, al entendimiento de que el mundo es dinámico y que el comercio global acelera éste cambio. En los últimos tiempos mucho se ha hablado de la política pública y el desarroIlo social, pero cuántos de aquellos manuscritos se han plasmado en mejores oportunidades para comunidades que aún son aisladas del desarrollo productivo -por ejemplo la Amazonía- , y precisamente son aisladas porque no disponen de infraestructura básica en comunicaciones, se debate sobre integración regional entre países que comparten relaciones comerciales, pero el único camino para lograr estos fines sobre los que mucha gente habla, es la infraestructura al servicio del transporte multimodal.

Descriptores: política pública, transporte multimodal, desarrollo local, integración regional.

\section{ABSTRACT}

The strategic importance of public policy with regard to the decision of investment in infrastructure for transportation service - but not the commonly known transport but that multimodal transport, planned and organized, that enhances the local development and encourages the integration of economic activities which take place in a country, as a crucial issue to secure the growth of the economies of the today's nations.

The issue of lack of infrastructure for multimodal transport has been a matter of political decision, rather than issues of financing these works, all for reasons opposite the changing, to the understanding that the world is dynamic and global trade accelerates this change. In recent years much has been said of public policy and social development, but how many of those manuscripts have ended in better opportunities for isolated communities are still insolated of productive development - for example the Amazon - and precisely because they are isolated these towns do not have the basic infrastructure in communications. There is a debate on regional integration among countries that share trade relations but the only way to achieve these goals for which many people talk about is having an infrastructure ready to work in the to multimodal transportation service.

Keywords: public policy, multimodal transport, local development, regional integration. 


\section{LA POLÍTICA PÚBLICA Y EL TRANSPORTE MULTIMODAL}

No se ha luchado contra la pobreza sin una infraestructura de conectividad, si la pobreza es marginada, la conectividad saca de la pobreza, por lo tanto los caminos sacan de la pobreza, pues las personas no tragarán más polvo, los productores pequeños podrán llegar con sus productos a mercados con mayores oportunidades, los niños podrán llegar más rápido a los centros de estudio, si hay una emergencia para la salud, se puede salir más rápido; hay una dimensión social, el agua potable rural, el alcantarillado rural, los by-pass en los pequeños pueblos para no destruirlos pasando por el medio, en fin; la política pública y el transporte multimodal son fundamentales para el desarrollo e integración regional de los países.

Bajo esta concepción existen tres grandes dimensiones: la productividad, competitividad y el beneficio social que genera la infraestructura al servicio del transporte multimodal y que van de la mano con el desarrollo económico e inclusión social.

Ahora bien, ¿Qué se entiende por transporte multimodal? Según la Convención de las Naciones Unidas sobre Transporte Multimodal de Mercancías, el Transporte Multimodal se define así: "El transporte de mercancía utilizando, al menos dos modos de transporte diferentes, cubierto por un solo contrato de transporte, desde un sitio en un país donde el operador de transporte multimodal se encarga de ellas, hasta un sitio designado para la entrega, situado en un país diferente"

Pero para lograr un transporte multimodal se necesita de la planificación del transporte, pues estudia las demandas presentes y futuras de movilidad de personas y materiales, precedidos por estudios de movimientos que necesariamente involucran a los diferentes medios de transporte. Está estrechamente relacionado con el campo de la ingeniería de tráfico (transporte). La planificación del transporte es la fase esencial del proceso de desarrollo y organización del mismo, pues es la que permite conocer los problemas, diseñar o crear soluciones $y$, en definitiva, optimizar y organizar los recursos para enfocarlos a atender la demanda de movilidad. En ella hay que destacar la importancia de asignar en los presupuestos los recursos necesarios para su realización.

La planificación del transporte regularmente ocurre en tres niveles:

\section{Planificación estatal}

Los departamentos de transporte deben desarrollar y mantener un proceso de planificación del trasporte multimodal en todo el sector, para lo cual se debe definir las necesidades de mejoramiento vial, y preparar planes regionales de transporte para múltiples sectores con fines también sociales.

\section{Planificación regional}

Las organizaciones de planeamiento sectorial son las que desarrollan sus propios planes regionales, que deben depender del proceso de planificación Estatal. El proceso de planificación regional requiere del desarrollo de un plan de largo alcance, el cual no solo define las necesidades regionales del transporte multimodal, sino que también identifica la fuente de fondos 
locales que se necesitarán para desarrollar los proyectos identificados.

\section{Planificación local}

La mayor parte de ciudades siguen un proceso similar de identificación de proyectos, costeo conceptual, y priorización de los caminos bajo su responsabilidad. Generalmente no son caminos bajo la responsabilidad de los departamentos de transporte estatales. Sin embargo, el Estado debe trabajar con las localidades para conseguir involucrar sus datos en un plan de largo alcance.

Entonces es de gran importancia la atención a estos lineamientos, ya que en la actualidad, el volumen de facturación generado por el transporte multimodal en la región Sudamericana - por citar un ejemplo asciende a 24 mil millones de dólares. Las perspectivas de incremento del comercio interregional son muy significativas para la región, pues existe un movimiento internacional de carga que asciende a 10 millones de TEUS por año, estableciéndose una tasa de crecimiento promedio anual del $8,8 \%$ en el comportamiento de la demanda futura. Por lo tanto, los países de la región experimentan un cambio dinámico en el comportamiento de sus variables macroeconómicas y las inversiones requeridas para atender dichos cambios, constituyen principalmente en la creación y mejoramiento de infraestructuras tanto físicas como administrativas para carga y pasajeros que se correlacionen con el desarrollo económico del territorio.
En el caso de la región amazónica, y más precisamente de las comunidades que no han sido atendidas efectivamente por la decisión política, desde muchos años atrás presenta graves problemas de integración por cuanto las condiciones geográficas son inhóspitas, pero presentan oportunidades para el desarrollo e integración regional.

Particularmente en la Amazonía que comparten Brasil, Colombia, Perú y Ecuador, en su cuenca central-occidental, la navegación fluvial es imprescindible: es la única forma de comunicación para una gran parte de los millones de ciudadanos que la habitan, es un componente central de la identidad amazónica, la cual es transversal a los pueblos amazónicos independientemente de su nacionalidad, es el modo de transporte más conveniente para el gran anhelo de preservación ambiental de la Amazonía, y es un excelente factor de integración ya que los ríos principales y los cientos de afluentes de distinta magnitud penetran la región a través de más de 20 mil kilómetros de vías navegables.

Es imprescindible realizar la planificación del transporte multimodal en la región amazónica que integre los aspectos físicos, jurídicos y operativos para lograr una gestión eficiente de la infraestructura, bajo una política pública de carácter global. 


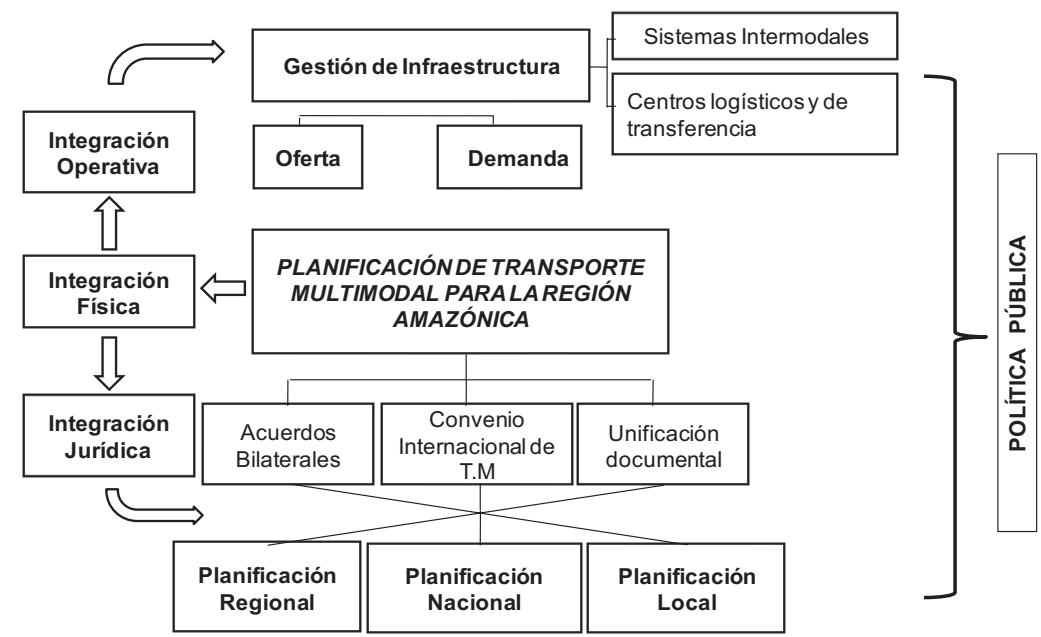

Elaboración: Autor

\section{Gráfico 1. Política pública y planificación del transporte multimodal}

El problema en común en la región amazónica, constituye la poca accesibilidad y la deficiente infraestructura en transporte, lo que hace que los costos sean elevados y como consecuencia, el encarecimiento de los productos. Los costos de mantenimiento de obras en la región amazónica, hacen que los proyectos de infraestructura transnacionales sean muy costosos. Por estas y otras razones, el transporte fluvial es el medio más apropiado para integrar a las zonas periféricas y generar desarrollo colectivo entre ellas.

La región amazónica es importante para el desarrollo de los países que la integran, pero la disponibilidad de infraestructura física para el transporte multimodal en la región amazónica es casi inexistente; si se toma en cuenta la oferta de servicios de transporte y de infraestructura necesaria para absorber el tráfico actual y el crecimiento potencial del transporte multimodal en la región presenta importantes deficiencias, unas más marcadas en unos países que en otros. Subsisten aún las políticas restrictivas a la entrada en los tráficos bilaterales y también prácticas de reserva de fletes, las cuales reducen artificialmente la oferta de los servicios de transporte, creando un aumento del precio de mercado y creando una tendencia a derivar el transporte multimodal en la región.

En las últimas décadas la región ha realizado un esfuerzo importante en alcanzar una más efectiva integración física. Especialmente en el último decenio se resalta la creación de la Red de Transporte y el inventario de Proyectos prioritarios para la integración de América del Sur, entre ella se encuentra la región amazónica. Es evidente que en la década de los noventa las estrategias de crecimiento consideraron a la integración regional como un complemento fundamental de sus propios mercados nacionales y, a la vez, una base necesaria para una eficiente inserción en los mercados internacionales con estructuras productivas eficaces. Resulta evidente también en los últimos años la importancia que va tomando el desarrollo de infraestructura de transportes en sus múltiples aspectos, que van desde el diseño, ejecución, mantenimiento de obras y prestación de múltiples servicios relacionados, hasta la 
misma planificación del transporte multimodal.

A grandes rasgos, la planificación del transporte en la región puede visualizarse a través de un sistema de corredores que, con algunas excepciones, se inician en los principales puertos, penetrando gradualmente al interior y conformando un subsistema multimodal de transporte regional que presenta la peculiaridad de conectar a los centros productores de la costa del Océano Atlántico y con los puertos en el Océano Pacífico, por ejemplo.

La política pública y el transporte multimodal tienen su incidencia en el desarrollo e integración regional ya que generan competitividad en tres frentes bien definidos: económicos, sociales y ambientales.

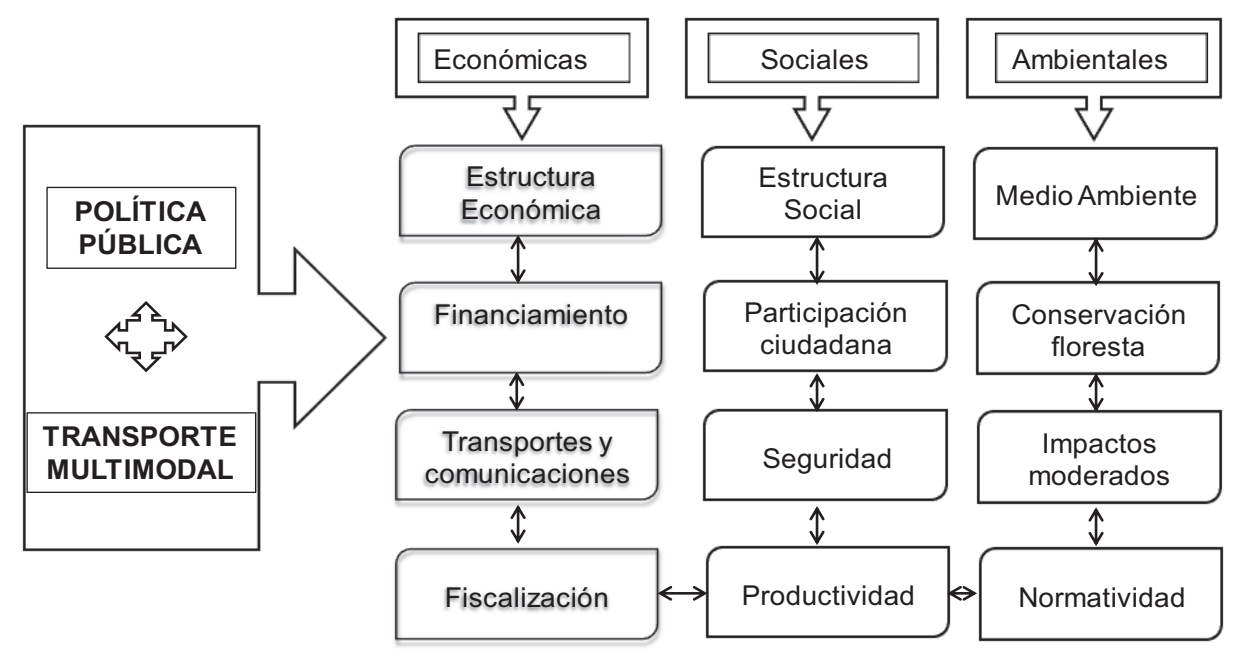

Elaboración: Autor

\section{Gráfico 2. Incidencia de la política pública y el transporte multimodal}

Asimismo, los proyectos de infraestructura que se cito anteriormente requieren elementos regulatorios por parte de la política pública ya que se suelen presentar monopolios en regiones donde la competencia es escasa, costos de financiamiento elevados, sistema de precios altos, sistemas indiscriminados de tributos, tiempos no razonables para recuperar inversiones, lo que agrava los aspectos de garantías y riesgos.
Por otra parte la misma política pública debe propiciar la participación del sector privado en proyectos de desarrollo e integración regional, lograr consecuencias económicas, sociales, geográficas y ambientales aceptables y minimizar costos $y$ riesgos a través de la confianza y logro de objetivos colectivos. 


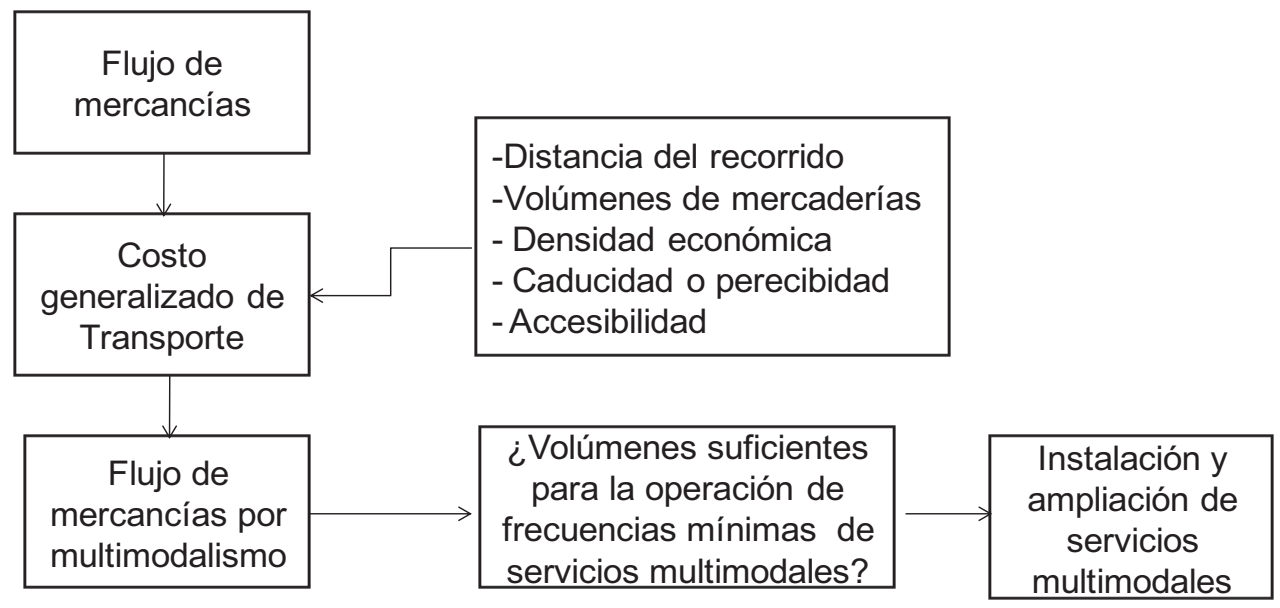

Elaboración: Autor

Gráfico 3. Proceso base para la planificación y competitividad

En la perspectiva del transporte multimodal, la planificación es fundamental pues permite conocer el flujo de mercaderías y personas que transitan por las vías, además permite realizar simulaciones de costos generalizados de viaje de acuerdo al tipo de transporte y ruta, ya que además de los fletes o costo de transporte entran en juego los llamados costos logísticos (costos de transferencia, costo del tiempo de espera, costo de servicios multimodales, costos externos, entre otros); y en base a esta planificación y análisis de resultados se estima volúmenes suficientes para la operación de frecuencias y los diferentes modos de transporte al servicio del multimodalismo.

El Plan Nacional para el Buen Vivir 2009 2013 planteado para Ecuador acoge una serie de reformas y reorganizaciones del Estado para concretar tareas que son necesidades de todos los ciudadanos, se determina un reordenamiento territorial (regiones) y a las cuales es necesario hacer converger la infraestructura a todo nivel, con el fin de que cada ciudadano sepa lo que tiene que hacer para apoyar al desarrollo del país, pero es también asunto de la credibilidad en la institucionalidad de las dependencias públicas y privadas que influyen en las estrategias para lograr los objetivos planteados en dicho Plan.

Es necesario saber cuál es la visión de país que se quiere dentro de 20 ó 30 años, para saber si lo que se está haciendo hoy es lo correcto en el futuro, pues el fenómeno de la globalización que empuja al mundo y aún más a un país como Ecuador hace que la economía sea dependiente de grandes economías y esto hace que los países en vías de desarrollo sirvan a la causa de las grandes potencias o primer mundo. Se ha firmado acuerdos internacionales con cantidad de países con el objetivo de mejorar el posicionamiento de la producción nacional y competitividad en el mercado internacional, las herramientas básicas de discusión son los aranceles y demás barreras al comercio; pero éste tema hace pensar en ¿qué tipo de infraestructura?, ¿qué sistemas de transporte?, ¿qué políticas?, entre otras, serán las más idóneas para con- 
tribuir a la competitividad del país en el comercio exterior y por eso es importante planificar para el futuro.

Cuántas de las carreteras existentes en el país no fueron diseñadas o pensadas para soportar el tráfico de hoy en día, mucho peor para después de 20 años.

Las cuestiones que ahora se plantean tienen que responderse de inmediato, bajo la planificación estratégica del transporte multimodal y para ello es necesario hacer adaptables las políticas públicas al desarrollo del país e integración regional. Asimismo, para que la política pública y el transporte multimodal logren un desarrollo efectivo es necesario hacer competitivas las actividades económicas que se realizan dentro del Ecuador, y es precisamente aquí donde el papel de los planificadores y demás actores involucrados en el desarrollo económico es preponderante.

\section{BIBLIOGRAFÍA}

ALBURQUERQUE, Francisco (1997). Desarrollo económico local y distribución del progreso técnico (Una respuesta a las exigencias del ajuste estructural) Cuadernos del ILPES 43, Santiago de Chile.

BARA, Neto Pedro; SÁNCHEZ, J. Ricardo; GORDON, Wilmsmeier (2005). Hacia un desarrollo sustentable e integrado de la Amazonía, división de recursos naturales e infraestructura, CEPAL, Comité de Coordinación Técnica IIRSA, Iniciativa para la Integración de la Infraestructura Regional Sudamericana, CEPAL/ONU - Corporación Andina de Fomento.

BOISIER, Sergio (1999). Política económica, organización social y desarrollo regional, Cuaderno 29, Serie II, Teoría y metáforas sobre desarrollo territorial.

CETIF-ECORAE (2003). Establecimiento de las directrices para el desarrollo del transporte intermodal en la región amazónica, Informe Final.

LIRA, Luis (2002), Documento de discusión interna ILPES, Preparado para la discusión conceptual sobre desarrollo territorial en el futuro Panorama de la Gestión Pública a someter a consideración de la XII Reunión del Consejo Regional de Planificación (CRP) del ILPES. 